\title{
Exponential stability of PI control for Saint-Venant equations with a friction term
}

\author{
Georges Bastin* and Jean-Michel Coron ${ }^{\dagger}$
}

Dedicated to Roland Glowinski, a master and a friend, on the occasion of his 80th birthday

\begin{abstract}
We consider open channels represented by Saint-Venant equations that are monitored and controlled at the downstream boundary and subject to unmeasured flow disturbances at the upstream boundary. We address the issue of feedback stabilization and disturbance rejection under Proportional-Integral (PI) boundary control. For channels with uniform steady states, the analysis has been carried out previously in the literature with spectral methods as well as with Lyapunov functions in Riemann coordinates. In this article, our main contribution is to show how the analysis can be extended to channels with non-uniform steady states with a Lyapunov function in physical coordinates.
\end{abstract}

\section{Introduction}

The hyperbolic Saint-Venant equations are commonly used for the description of water flow dynamics in open channels and for the design of management and control systems in irrigation networks and navigable rivers. In particular, the exponential stabilization of Saint-Venant equations by boundary feedback control has been a recurring research topic in the literature for more than twenty years.

The earlier results dealt with static proportional control. In the simplest case of horizontal channels with negligible friction, the stability analysis was carried out in [6] with an entropy Lyapunov function, in [16, 11] with the method of characteristics, and in [7, Section VI] with a Lyapunov function in Riemann coordinates. The stability analysis was then extended to channels with slope and friction. In the special case of a uniform steady state, the stability analysis was carried out with a spectral method for linearized equations in [17, Section 6]. However the linearized system stability does not directly imply the stability of the steady state for the nonlinear SaintVenant equations (see e.g. [8]). For this nonlinear case, the stability analysis is done in [4, 13] with a Lyapunov function in Riemann coordinates. More recently, the case of channels with friction and slope and non-uniform steady state was considered in [3] and [15] with dedicated Lyapunov functions expressed in physical coordinates.

The boundary feedback stabilization of Saint-Venant equations by Proportional-Integral (PI) control has received much less attention in the literature. It has been analyzed for channels with uniform steady states in [5] with a spectral method and in [14, Section 4], [2, Section 5.5] with Lyapunov functions in Riemann coordinates. In the present article, our main contribution is to show how the analysis of [3] can be extended to channels with non-uniform steady states under PI control, using a Lyapunov function in physical coordinates.

Obviously, in principle, stabilization is also possible with more sophisticated control laws. In particular, the recent backstepping method for $2 \times 2$ hyperbolic systems, see e.g. [10, 1, 12,

\footnotetext{
*Department of Mathematical Engineering, ICTEAM, University of Louvain, Louvain-La-Neuve, Belgium.

†Sorbonne Université, Université Paris-Diderot SPC, CNRS, INRIA, Laboratoire Jacques-Louis Lions, LJLL, équipe CAGE, F-75005 Paris, France.
} 
allows to design stabilizing boundary output feedbacks in observer-controller form for Saint-Venant equations. However, it is clear that such advanced solutions are far from being used in practice and that PI controllers are the only regulators that are really implemented in the vast majority of field applications. The reason is obviously that PI regulators, besides their great ease of implementation, are the simplest solution to cancel off-set errors and attenuate load disturbances. In a PI regulator, the parameter $k_{i}$ is a measure of the disturbance attenuation efficiency, but too large values may produce instability. The analysis of the stability of a closed-loop system under PI control, as we present in this article, is therefore an important and relevant issue.

\section{Saint-Venant equations}

We consider a pool of a prismatic horizontal open channel with a rectangular cross section, as shown in Fig 1. The dynamics of the system are described by the Saint-Venant equations

$$
\begin{aligned}
& H_{t}+(H V)_{x}=0, \\
& V_{t}+\left(g H+\frac{1}{2} V^{2}\right)_{x}+S_{f}(H, V)=0,
\end{aligned}
$$

with the state variables $H(t, x)=$ water depth and $V(t, x)=$ horizontal water velocity at the time instant $t$ and the location $x$ along the channel. $L$ is the length of the pool and $g$ is the gravity acceleration. $S_{f}(H, V)$ is the friction term for which various empirical models are available in the engineering literature. In this article, we adopt the simple model

$$
S_{f}(H, V) \triangleq C \frac{V^{2}}{H}
$$

with $C$ a constant friction coefficient.

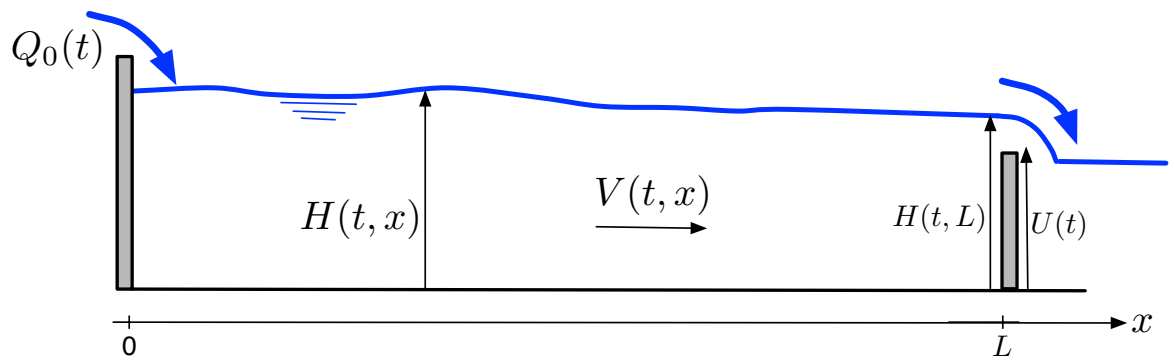

Figure 1: Pool of an open channel with an overflow gate at the downstream side.

The system is subject to the following boundary conditions:

$$
\begin{aligned}
& H(t, 0) V(t, 0)=Q_{0}(t), \\
& H(t, L) V(t, L)=v_{G}(H(t, L)-U(t)) .
\end{aligned}
$$

The first boundary condition (3a) imposes the value of the canal inflow rate which is an unknown disturbance denoted $Q_{0}(t)$. The second boundary condition $(3 \mathrm{~b})$ is a simple linear model of a spillway outflow gate with $U(t)$ the gate elevation used as control input and $v_{G}$ a constant gate parameter. 


\section{Proportional-Integral control}

In this article we are concerned with the case where the outflow gate is provided with a ProportionalIntegral (PI) control law

$$
U(t) \triangleq U_{r}+k_{p}\left(H_{s p}-H(t, L)\right)+k_{i} \int_{0}^{t}\left(H_{s p}-H(\tau, L)\right) d \tau
$$

where $H_{s p}$ denotes the set-point for the downstream level $H(t, L)$ which is assumed to be measured on line. The first term $U_{r}$ is an arbitrary constant value for the gate elevation. The second term is the proportional correction action with the tuning parameter $k_{p}$. The last term is the integral action with the tuning parameter $k_{i}$.

With this control law, defining $Z(t) \triangleq U(t)+k_{p} H(t, L)$, the boundary conditions are written in differential form as follows:

$$
\begin{aligned}
& H(t, 0) V(t, 0)=Q_{0}(t), \\
& H(t, L) V(t, L)=v_{G}\left[\left(1+k_{p}\right) H(t, L)-Z(t)\right], \\
& \frac{d Z}{d t}=k_{i}\left(H_{s p}-H(t, L)\right) .
\end{aligned}
$$

When $U(t)$ is the feedback command signal (4), the system (1), (5) is a closed loop boundary control system.

In this article, our main purpose is to analyze the exponential stability of this closed loop control system.

Remark 1. It would be interesting to know if, as in the case of a single linear transport equation (see [9, Theorem 2.2]), the control system (1) subject to Proportional-Integral-Derivative (PID) boundary controls is always unstable.

\section{Fluvial steady state}

In case of a constant positive disturbance $Q_{0}>0$ and a constant positive set point $H_{s p}>0$, a steady state of the closed loop control system is a time-invariant solution $H^{*}(x), V^{*}(x), Z^{*}$, $x \in[0, L]$, given by:

$$
\begin{aligned}
& H^{*}(x) \text { solution of }\left(g H^{* 3}-Q_{0}^{2}\right) H_{x}^{*}+C Q_{0}^{2}=0, H^{*}(L)=H_{s p}, \\
& V^{*}(x)=\frac{Q_{0}}{H^{*}(x)}, \\
& Z^{*}=\left(1+k_{p}\right) H_{s p}-\frac{Q_{0}}{v_{G}} .
\end{aligned}
$$

The existence of a solution to (6a) requires that $g H_{s p}^{3} \neq Q_{0}^{2}$. If $g H_{s p}^{3}>Q_{0}^{2}$, then 6a has a solution (note that $H^{*}$ is then decreasing) and the steady state flow is subcritical (or fluvial). In such case, from (6a) and (6b), according to the physical evidence, the state $\left(H^{*}, V^{*}\right)$ is positive :

$$
H^{*}(x)>0, \quad V^{*}(x)>0, \text { for all } x \in[0, L],
$$

and satisfies the following inequality:

$$
0<g H^{*}(x)-V^{* 2}(x)=g H^{* 3}(x)-Q_{0}^{2}, \quad \forall x \in[0, L] .
$$

In the case where $g H_{s p}^{3}<Q_{0}^{2}$, the steady state, if it exists, is said to be supercritical (or torrential). We do not consider that case in the present article. 


\section{Linearization}

In order to linearize the model, we define the deviations of the states $H(t, x), V(t, x)$ and $Z(t)$ with respect to the steady states $H^{*}(x), V^{*}(x)$ and $Z^{*}$ :

$$
h(t, x) \triangleq H(t, x)-H^{*}(x), \quad v(t, x) \triangleq V(t, x)-V^{*}(x), \quad z=Z(t)-Z^{*} .
$$

Then the linearized Saint-Venant equations around the steady-state are

$$
\left(\begin{array}{c}
h_{t} \\
v_{t}
\end{array}\right)+\left(\begin{array}{cc}
V^{*} & H^{*} \\
g & V^{*}
\end{array}\right)\left(\begin{array}{l}
h_{x} \\
v_{x}
\end{array}\right)+\left(\begin{array}{cc}
V_{x}^{*} & H_{x}^{*} \\
-C \frac{V^{* 2}}{H^{* 2}} & V_{x}^{*}+2 C \frac{V^{*}}{H^{*}}
\end{array}\right)\left(\begin{array}{l}
h \\
v
\end{array}\right)=0
$$

and the linearized boundary conditions are

$$
\begin{aligned}
& v(t, 0)=-b_{0} h(t, 0) \quad \text { with } \quad b_{0}=\frac{V^{*}(0)}{H^{*}(0)}, \\
& v(t, L)=b_{L} h(t, L)-b_{z} z(t), \quad b_{L}=\frac{v_{G}\left(1+k_{p}\right)-V^{*}(L)}{H^{*}(L)}, \quad b_{z}=\frac{v_{G}}{H^{*}(L)}, \\
& z_{t}=-k_{i} h(t, L) .
\end{aligned}
$$

\section{Exponential stability of the linearized system}

Let us consider the linearized system (10), 111) under an initial condition

$$
h(0, x)=h^{o}(x), \quad v(0, x)=v^{o}(x), \quad z(0)=z^{o},
$$

such that

$$
\left(h^{o}, v^{o}\right) \in L^{2}\left((0, L) ; \mathbb{R}^{2}\right), \quad z^{o} \in \mathbb{R} .
$$

The Cauchy problem (10)-111)-12 is well-posed (see [2, Appendix A]).

Our concern is to analyze the exponential stability of the system (10)-(11) according to the following definition.

Definition 1. The system (10)-(11) is exponentially stable (for the $L^{2}$-norm) if there exist $\nu>0$ and $C_{o}>0$ such that, for every initial condition $\left(h^{o}, v^{o}\right) \in L^{2}\left((0, L) ; \mathbb{R}^{2}\right), z^{o} \in \mathbb{R}$, the solution to the Cauchy problem 10, 111, 12, satisfies

$$
\|(h(t, \cdot), v(t, \cdot))\|_{L^{2}}+|z(t)| \leqslant C_{o} e^{-\nu t}\left[\left\|\left(h^{o}, v^{o}\right)\right\|_{L^{2}}+\left|z^{o}\right|\right] .
$$

We now prove that the linearized control system (10)- 11 is exponentially stable if the steady state is subcritical and the control tuning parameters are positive: $k_{p}>0$ and $k_{i}>0$. For this stability analysis, the following candidate Lyapunov function is considered:

$$
\mathbf{V}(h, v, z)=\int_{0}^{L}\left(g h^{2}+H^{*} v^{2}\right) d x+q z^{2},
$$

where $q>0$ is chosen later on. Note that there exists $C_{1}>0$ such that

$$
\frac{1}{C_{1}}\left(\|(h, v)\|_{L^{2}}^{2}+|z|^{2}\right) \leq \mathbf{V}(h, v, z) \leq C_{1}\|(h, v)\|_{L^{2}}^{2}+|z|^{2}, \quad \forall(h, v, z) \in L^{2}(0, L) \times L^{2}(0, L) \times \mathbb{R} .
$$

The time derivative of this function $\mathbf{V}$ along the $C^{1}$ solutions of the Cauchy problem $[10$, , 11, (12) is

$$
\frac{d \mathbf{V}}{d t}=2 \int_{0}^{L}\left(g h h_{t}+H^{*} v v_{t}\right) d x+2 q z z_{t} .
$$


Using the system equation 10 and the boundary condition $11 \mathrm{c}$, we have

$$
\begin{aligned}
\frac{d \mathbf{V}}{d t}=-2 \int_{0}^{L}\left(g h\left(V^{*} h_{x}+H^{*} v_{x}+V_{x}^{*} h+H_{x}^{*} v\right)\right. & \\
& \left.+H^{*} v\left(g h_{x}+V^{*} v_{x}-C \frac{V^{* 2}}{H^{* 2}} h+\left(V_{x}^{*}+2 C \frac{V^{*}}{H^{*}} v\right)\right)\right) d x-2 q k_{i} z h(t, L) .
\end{aligned}
$$

Then, using integration by parts together with $6 \mathrm{a}$, we have

$$
\left.\frac{d \mathbf{V}}{d t}=-\left[\begin{array}{ll}
h & v
\end{array}\right) M(x)\left(\begin{array}{l}
h \\
v
\end{array}\right)\right]_{0}^{L}-\int_{0}^{L}\left(\begin{array}{ll}
h & v
\end{array}\right) N(x)\left(\begin{array}{l}
h \\
v
\end{array}\right) d x-2 q k_{i} z h(t, L),
$$

with

$$
M(x)=\left(\begin{array}{cc}
g V^{*}(x) & g H^{*}(x) \\
g H^{*}(x) & H^{*}(x) V^{*}(x)
\end{array}\right)
$$

and

$$
N(x)=\left(\begin{array}{cc}
\frac{g C V^{* 3}}{H^{*}\left(g H^{*}-V^{* 2}\right)} & -\frac{C V^{* 2}}{H^{*}} \\
-\frac{C V^{* 2}}{H^{*}} & \frac{2 C V^{* 3}}{\left(g H^{*}-V^{* 2}\right)}+4 C V^{*}
\end{array}\right) .
$$

We introduce the notations

$$
\begin{aligned}
h_{0} & =h(t, 0), \quad h_{L}=h(t, L), \quad v_{0}=v(t, 0), \quad v_{L}=v(t, L), \\
H_{0} & =H^{*}(0), \quad V_{0}=V^{*}(0), \quad H_{L}=H^{*}(L), \quad V_{L}=V^{*}(L) .
\end{aligned}
$$

Then, using the boundary conditions 11a, 11b, we have

$$
\begin{aligned}
& {\left[\left(\begin{array}{ll}
h & v
\end{array}\right) M(x)\left(\begin{array}{l}
h \\
v
\end{array}\right)\right]_{0}^{L}=g V_{L} h_{L}^{2}+2 g H_{L} h_{L} v_{L}+Q_{0} v_{L}^{2}-g V_{0} h_{0}^{2}-2 g H_{0} h_{0} v_{0}-Q_{0} v_{0}^{2}} \\
& =g V_{L} h_{L}^{2}+2 g H_{L} h_{L}\left(b_{L} h_{L}-b_{z} z\right)+Q_{0}\left(b_{L} h_{L}-b_{z} z\right)^{2}-g V_{0} h_{0}^{2}+2 g H_{0} h_{0}\left(b_{0} h_{0}\right)-Q_{0}\left(b_{0} h_{0}\right)^{2} \\
& =\left(g V_{L}+2 g b_{L} H_{L}+Q_{0} b_{L}^{2}\right) h_{L}^{2}+\left(-g V_{0}+2 g b_{0} H_{0}-Q_{0} b_{0}^{2}\right) h_{0}^{2}+Q_{0} b_{z}^{2} z^{2} \\
& +\left(-2 g b_{z} H_{L}-2 Q_{0} b_{L} b_{z}\right) h_{L} z .
\end{aligned}
$$

Consequently

$$
\frac{d \mathbf{V}}{d t}=-m_{0} h_{0}^{2}-\left(\begin{array}{ll}
h_{L} & z
\end{array}\right) \mathcal{M}\left(\begin{array}{c}
h_{L} \\
z
\end{array}\right)-\int_{0}^{L}\left(\begin{array}{ll}
h & v
\end{array}\right) N(x)\left(\begin{array}{l}
h \\
v
\end{array}\right) d x-2 a q k_{i} z^{2},
$$

with

$$
\begin{gathered}
m_{0}=-g V_{0}+2 g b_{0} H_{0}-Q_{0} b_{0}^{2}, \\
\mathcal{M}=\left(\begin{array}{cc}
g V_{L}+2 g b_{L} H_{L}+Q_{0} b_{L}^{2} & -g b_{z} H_{L}-Q_{0} b_{L} b_{z}+q k_{i} \\
-g b_{z} H_{L}-Q_{0} b_{L} b_{z}+q k_{i} & Q_{0} b_{z}^{2}-2 a q k_{i}
\end{array}\right),
\end{gathered}
$$

and $a$ is a real positive constant to be determined.

Under the subcritical flow condition (8), using the definition of $b_{0}$ (11a), we have that

$$
m_{0}=-g V_{0}+2 g b_{0} H_{0}-H_{0} V_{0} b_{0}^{2}=b_{0}\left(g H_{0}-V_{0}^{2}\right)>0
$$


and that the matrix $N(x)$ is positive definite for all $x \in[0, L]$ since

$$
\operatorname{det}[N(x)]=\left(\frac{C V^{* 2}}{H^{*}}\right)^{2}\left(\frac{2 g H^{*} V^{* 2}}{\left(g H^{*}-V^{* 2}\right)^{2}}+\frac{4 g H^{*}}{\left(g H^{*}-V^{* 2}\right)}-1\right)>0 .
$$

On the other hand, $\mathcal{M}$ is positive definite if

(a) $g V_{L}+2 g b_{L} H_{L}+Q_{0} b_{L}^{2}>0$,

(b) $\operatorname{det}(\mathcal{M})=\left(g V_{L}+2 g b_{L} H_{L}+Q_{0} b_{L}^{2}\right)\left(Q_{0} b_{z}^{2}-2 a q k_{i}\right)-\left(g b_{z} H_{L}+Q_{0} b_{L} b_{z}-q k_{i}\right)^{2}>0$.

It follows from (3b) that $v_{G}>V_{L}$. Hence, since $k_{p}>0$, we have from 11b

$$
b_{L}=\frac{\left(v_{G}-V_{L}\right)+v_{G} k_{p}}{H^{*}(L)}>0
$$

and Condition (a) is satisfied.

Regarding condition (b), using the definition of $b_{L}$, we have

$$
\operatorname{det}(\mathcal{M})=-\alpha+2 \beta k_{i} q-k_{i}^{2} q^{2}=\mathcal{P}(q),
$$

with

$$
\alpha=g b_{z}^{2} H_{L}\left(g H_{L}-V_{L}^{2}\right)
$$

and

$$
\beta=g b_{z} H_{L}+Q_{0} b_{L} b_{z}-a\left(g V_{L}+2 g b_{L} H_{L}+Q_{0} b_{L}^{2}\right) .
$$

$\mathcal{P}(q)$ is a degree-2 polynomial in $q$ with discriminant

$$
\Delta=4 k_{i}^{2}\left(\beta^{2}-\alpha\right) .
$$

We observe that $\alpha>0$ under the subcritical flow condition (8). Moreover, it is easy to check that the positive parameter $a$ can be selected sufficiently small so that $\beta>0$ and $\beta^{2}-\alpha>0$. Hence, if $k_{i}>0, \mathcal{P}(q)$ has two positive real roots and there exists a positive value of $q$ (depending on $k_{i}$ ) such that $\operatorname{det}(\mathcal{M})>0$ and condition (b) is satisfied. Then, it follows directly from the definition (15) of $\mathbf{V}$ and from (25) that there exists a positive real constant $\mu$ such that

$$
\frac{d \mathbf{V}}{d t} \leqslant-\mu \mathbf{V}
$$

along the $C^{1}$-solutions of the system. However, since the $C^{1}$-solutions are dense in the set of $L^{2}$-solutions, inequality (37) is also satisfied in the sense of distributions for $L^{2}$-solutions (see [2] for details). Consequently, $\mathbf{V}$ is an exponentially decaying Lyapunov function for the $L^{2}$-norm and the system (10)-11) is exponentially stable in the sense of Definition 1.

\section{Exponential stability of the steady state of the Saint-Venant equations}

In the previous section, we have shown that the PI controller (4) stabilizes the linearized SaintVenant equations if the steady state is subcritical and the control tuning parameters are positive: $k_{p}>0$ and $k_{i}>0$. In this section, we briefly explain how it can be shown that the same PI controller is also sufficient to guarantee the local exponential stability for the $H^{2}$-norm of the steady state $H^{*}(x), V^{*}(x)$ of the nonlinear system of Saint-Venant equations (1), (2) under the nonlinear boundary conditions $(5)$. 
Let us rewrite the Saint-Venant equations in the $(h, v)$ coordinates (see $(9)$ ),

$$
\begin{aligned}
& \left(\begin{array}{l}
h_{t} \\
v_{t}
\end{array}\right)+\left(\begin{array}{cc}
V^{*}(x)+v & H^{*}(x)+h \\
g & V^{*}(x)+v
\end{array}\right)\left(\begin{array}{l}
h_{x} \\
v_{x}
\end{array}\right) \\
& +\left(\begin{array}{cc}
V_{x}^{*}(x) & H_{x}^{*}(x) \\
-C \frac{V^{* 2}(x)}{H^{*}(x)\left(H^{*}(x)+h\right)} & V_{x}^{*}(x)+C \frac{2 V^{*}(x)+v}{H^{*}(x)+h}
\end{array}\right)\left(\begin{array}{l}
h \\
v
\end{array}\right)=0,
\end{aligned}
$$

with the boundary conditions (using the notations 22 and $(23)$

$$
\begin{aligned}
& v_{0}=-b_{0} h_{0}+\frac{V_{0}}{H_{0}\left(H_{0}+h_{0}\right)} h_{0}^{2}, \\
& v_{L}=b_{L} h_{L}-b_{z} z+\frac{\left(V_{L}-v_{G}\left(1+k_{P}\right)\right) h_{L}^{2}+v_{G} h_{L} z}{H_{L}\left(H_{L}+h_{L}\right)}, \\
& z_{t}=-k_{i} h_{L} .
\end{aligned}
$$

Then, we transform the system into Riemann coordinates which are defined as follows:

$$
\mathbf{R}=\left(\begin{array}{l}
R^{+} \\
R^{-}
\end{array}\right)=\left(\begin{array}{l}
v+2 \eta(h) \\
v-2 \eta(h)
\end{array}\right) \text { with } \eta(h)=\sqrt{g\left(H^{*}+h\right)}-\sqrt{g H^{*}} .
$$

With these coordinates, the system $[38)$ is written in the following characteristic form:

$$
\mathbf{R}_{t}+\Lambda(\mathbf{R}, x) \mathbf{R}_{x}+\mathbf{B}(\mathbf{R}, x)=\mathbf{0}
$$

with the diagonal matrix

$$
\Lambda(\mathbf{R}, x)=\left(\begin{array}{cc}
\lambda^{+}(\mathbf{R}, x) & 0 \\
0 & \lambda^{-}(\mathbf{R}, x)
\end{array}\right) \quad \text { with } \lambda^{ \pm}(\mathbf{R}, x)=V^{*} \pm \sqrt{g H^{*}}+v \pm \eta(h),
$$

and an appropriate definition of $\mathbf{B}(\mathbf{R}, x)$.

The goal is to prove the $H^{2}$ exponential stability of the zero steady state for the system 42 under the boundary conditions $(39)$ and under an initial condition

$$
\mathbf{R}(0, x)=\mathbf{R}_{\mathrm{o}}(x), \quad z(0)=z^{o} .
$$

according to the following definition.

Definition 2. The steady state $\mathbf{R}(t, x) \equiv 0$ of the system 39 and 41 is exponentially stable for the $H^{2}$-norm if there exist $\delta>0, \nu>0$ and $C_{0}>0$ such that, for every initial condition

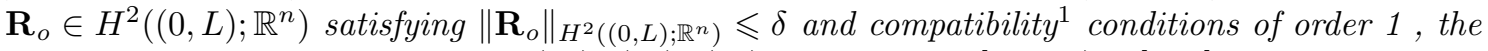
solution $\mathbf{R}$ of the Cauchy problem (39), (41), (43) is defined on $[0,+\infty) \times[0, L]$ and satisfies

$$
\|\mathbf{R}(t, .)\|_{H^{2}\left((0, L) ; \mathbb{R}^{n}\right)}+|z(t)| \leq C_{0} e^{-\nu t}\left[\left\|\mathbf{R}_{o}\right\|_{H^{2}\left((0, L) ; \mathbb{R}^{n}\right)}+\left|z^{o}\right|\right] .
$$

With this definition our main result is the following theorem

Theorem 1. The steady state $\mathbf{R}(t, x) \equiv 0$ of the system (39) and 41) is exponentially stable for the $H^{2}$-norm.

\footnotetext{
${ }^{1}$ For an explanation of the concept of compatibility of initial conditions, see [2, Section 4.5.2]
} 
The proof of Theorem 1 is built in a way similar to the proof given in [2, Chapter 6] for a general class of quasi-linear hyperbolic systems with static boundary conditions. Here we limit ourselves to the key points of the proof and we refer the reader to [2, Section 6.2] for a comprehensive development.

First, we consider an augmented system with state $\left(\mathbf{R}, \mathbf{R}_{t}, \mathbf{R}_{t t}\right)$ where the dynamics of $\mathbf{R}_{t}$ and $\mathbf{R}_{t t}$ are simply obtained by taking partial time derivatives of the system equation (42) and the boundary conditions 39 .

Then the candidate Lyapunov function is defined as

$$
\mathbf{V}_{\mathrm{NL}}=\mathbf{V}_{\mathbf{1}}(\mathbf{R}, z)+\mathbf{V}_{\mathbf{2}}\left(\mathbf{R}_{t}, z_{t}\right)+\mathbf{V}_{\mathbf{3}}\left(\mathbf{R}_{t t}, z_{t t}\right)
$$

with

$$
\begin{gathered}
\mathbf{V}_{\mathbf{1}}(\mathbf{R}, z)=\int_{0}^{L} \frac{1}{2} H^{*} \mathbf{R}^{T} \mathbf{R} d x+q z^{2}, \\
\mathbf{V}_{\mathbf{2}}\left(\mathbf{R}_{t}, z_{t}\right)=\int_{0}^{L} \frac{1}{2} H^{*} \mathbf{R}_{t}^{T} \mathbf{R}_{t} d x+q z_{t}^{2}, \\
\mathbf{V}_{\mathbf{3}}\left(\mathbf{R}_{t t}, z_{t t}\right)=\int_{0}^{L} \frac{1}{2} H^{*} \mathbf{R}_{t t}^{T} \mathbf{R}_{t t} d x+q z_{t t}^{2} .
\end{gathered}
$$

For a vector $\boldsymbol{\xi}=\left(\xi_{1}, \ldots, \xi_{n}\right)^{T} \in \mathbb{R}^{n}$, we denote $|\boldsymbol{\xi}|_{\infty}=\max \left\{\left|\xi_{j}\right| ; j \in\{1, \ldots, n\}\right\}$. For a map $f \in C^{0}\left([0, L] ; \mathbb{R}^{n}\right)$, we denote $|f|_{0}=\max \left\{|f(x)|_{\infty} ; x \in[0, L]\right\}$. We remark that, for small $|h|_{0}$, the function $\mathbf{V}_{\mathbf{1}}(\mathbf{R}, z)$ can be viewed as a perturbation of the Lyapunov function $\mathbf{V}(h, v, z)$ of the linearized system (see equation $(15)$ ). More precisely, for $|h|_{0}$ sufficiently small,

$$
\begin{aligned}
\mathbf{V}_{\mathbf{1}}(\mathbf{R}, z) & =\int_{0}^{L} \frac{1}{2} H^{*} \mathbf{R}^{T} \mathbf{R} d x+q z^{2} \\
& =\int_{0}^{L}\left(4 H^{*} \eta^{2}(h)+H^{*} v^{2}\right) d x+q z^{2} \\
& =\int_{0}^{L}\left(g h^{2}+H^{*} v^{2}+O\left(h^{3}\right)\right) d x+q z^{2} \\
& =\mathbf{V}(h, v, z)+\int_{0}^{L} O\left(|h|^{3}\right) d x
\end{aligned}
$$

Similar expressions of $\mathbf{V}_{\mathbf{2}}$ and $\mathbf{V}_{\mathbf{3}}$ are obtained as follows: for $|h|_{0}$ sufficiently small

$$
\begin{gathered}
\mathbf{V}_{\mathbf{2}}\left(\mathbf{R}_{t}, z_{t}\right)=\mathbf{V}\left(h_{t}, v_{t}, z_{t}\right)+\int_{0}^{L} O\left(\left|h h_{t}^{2}\right|\right) d x \\
\mathbf{V}_{\mathbf{3}}\left(\mathbf{R}_{t t}, z_{t t}\right)=\mathbf{V}\left(h_{t t}, v_{t t}, z_{t t}\right)+\int_{0}^{L} O\left(\left|h_{t}^{2} h_{t t}\right|+\left|h h_{t t}^{2}\right|+\left|h_{t}\right|^{4}\right) d x .
\end{gathered}
$$

Let us now introduce a notation to deal with "higher order terms" in the time derivative of the Lyapunov function. We denote by $\mathcal{O}\left(X_{1} ; X_{2}\right)$, with $X_{1} \geqslant 0$ and $X_{2} \geqslant 0$, quantities for which there exist $C_{0}>0$ and $\varepsilon>0$ independent of $\mathbf{R}, \mathbf{R}_{t}$ and $\mathbf{R}_{t t}$, such that

$$
\left(X_{2} \leqslant \varepsilon\right) \Longrightarrow\left(\left|\mathcal{O}\left(X_{1} ; X_{2}\right)\right| \leqslant C_{0} X_{1}\right) .
$$

It follows that the time derivatives of $\mathbf{V}_{\mathbf{1}}, \mathbf{V}_{\mathbf{2}}$ and $\mathbf{V}_{\mathbf{3}}$ along the system solutions can be expressed 
in the following form

$$
\begin{array}{r}
\left.\frac{d \mathbf{V}_{\mathbf{1}}}{d t}=-\left[\begin{array}{ll}
h & v
\end{array}\right) M(x)\left(\begin{array}{l}
h \\
v
\end{array}\right)\right]_{0}^{L}-\int_{0}^{L}\left(\begin{array}{ll}
h & v
\end{array}\right) \\
+\mathcal{O}\left(|\mathbf{R}(t, 0)|^{3}+|\mathbf{R}(t, L)|^{3} ;|\mathbf{R}(t, 0)|+|\mathbf{R}(t, L)|\right) \\
v \\
+\mathcal{O}\left(\int_{0}^{L}\left(|\mathbf{R}|^{3}+\left|\mathbf{R}_{t}\right||\mathbf{R}|^{2}\right) d x ;|\mathbf{R}(t, .)|_{0}\right)
\end{array}
$$

$$
\begin{array}{r}
\left.\frac{d \mathbf{V}_{\mathbf{2}}}{d t}=-\left[\begin{array}{ll}
\left(h_{t}\right. & v_{t}
\end{array}\right) M(x)\left(\begin{array}{c}
h_{t} \\
v_{t}
\end{array}\right)\right]_{0}^{L}-\int_{0}^{L}\left(\begin{array}{ll}
h_{t} & v_{t}
\end{array}\right) N(x)\left(\begin{array}{c}
h_{t} \\
v_{t}
\end{array}\right) d x-2 q z_{t} z_{t t} \\
\left.+\left|\mathbf{R}_{t}(t, 0)\right|^{2}|\mathbf{R}(t, 0)|+\left|\mathbf{R}_{t}(t, L)\right|^{2}|\mathbf{R}(t, L)| ;|\mathbf{R}(t, 0)|+|\mathbf{R}(t, L)|\right) \\
\left.+\mathcal{O}\left(\int_{0}^{L}\left|\mathbf{R}_{t}\right|^{2}\left(\left|\mathbf{R}_{t}\right|+|\mathbf{R}|\right) d x ;|\mathbf{R}(t, .)|_{0}\right)\right),
\end{array}
$$

$$
\begin{aligned}
\frac{d \mathbf{V}_{3}}{d t}=-\left[\left(\begin{array}{ll}
h_{t t} & v_{t t}
\end{array}\right) M(x)\left(\begin{array}{c}
h_{t t} \\
v_{t t}
\end{array}\right)\right]_{0}^{L}-\int_{0}^{L}\left(\begin{array}{ll}
h_{t} & v_{t}
\end{array}\right) N(x)\left(\begin{array}{c}
h_{t} \\
v_{t}
\end{array}\right) d x-2 q z_{t t} z_{t t t} \\
+ \\
+\left|\mathbf{O}_{t t}(t, L)\right|^{2}|\mathbf{R}(t, L)|+\left|\mathbf{R}_{t t}(t, 0)\right|^{2}|\mathbf{R}(t, 0)|+\left|\mathbf{R}_{t t}(t, 0)\right|\left|\mathbf{R}_{t}(t, 0)\right|^{2}+\left|\mathbf{R}_{t}(t, 0)\right|^{4} \\
+ \\
+\mathcal{O}\left(\int_{0}^{L}\left(\left|\mathbf{R}_{t t}(t, L)\right|^{2}\left(\left|\mathbf{R}_{t}\right|+|\mathbf{R}|\right)+\left|\mathbf{R}_{t}(t, L)\right|^{4} ;|\mathbf{R}(t, 0)|+|\mathbf{R}(t, L)|\right)\right.
\end{aligned}
$$

We observe that, in each case, we recover the quadratic formula of the linear case augmented with (at least) cubic terms that are negligible for small $|\mathbf{R}(t, .)|_{0}+\left|\mathbf{R}_{t}(t, .)\right|_{0}$. It is therefore not surprising that the local $H^{2}$ stability of the nonlinear steady state can be deduced from the global $L^{2}$ stability of the linear system. By proceeding similarly to [2, Chapter 6], it can be shown that there exist positive constants $\alpha$ and $\delta$ such that, for every $\mathbf{R}$ such that $|\mathbf{R}(t, .)|_{0}+\left|\mathbf{R}_{t}(t, .)\right|_{0}<\delta$, we have

$$
\frac{d \mathbf{V}_{\mathrm{NL}}}{d t} \leqslant-\alpha \mathbf{V}_{\mathrm{NL}}
$$

along the system solutions. Compared to [2, Chapter 6], note that, by (39b), $v_{L}$ (and its first and second time derivatives) can be expressed in terms of $h_{L}$ and $z$ (and their first and second time derivatives) as we did in (24) for the linear case. It follows that the system steady-state is locally exponentially stable for the $H^{2}$-norm in the sense of Definition 2. This concludes the proof of Theorem 1.

\section{Conclusion}

In this article, our main contribution was to exhibit a Lyapunov function which allows to study the exponential stability of nonuniform steady-states for Saint-Venant equations with a friction term under boundary feedback PI control.

Acknowledgments. The two authors thank Amaury Hayat for useful comments. They also thank ETH-ITS and ETH-FIM for their hospitality. This work was partly completed during their stay in these two institutions. Both authors were supported by ANR Project Finite4SoS (ANR 15-CE23-0007). 


\section{References}

[1] Henrik Anfinsen and Ole Morten Aamo. Adaptive output-feedback stabilization of linear $2 \times 2$ hyperbolic systems using anti-collocated sensing and control. Systems Control Lett., 104:86-94, 2017.

[2] Georges Bastin and Jean-Michel Coron. Stability and Boundary Stabilisation of 1-D Hyperbolic Systems. Number 88 in Progress in Nonlinear Differential Equations and Their Applications. Springer International, 2016.

[3] Georges Bastin and Jean-Michel Coron. A quadratic Lyapunov function for hyperbolic density-velocity systems with nonuniform steady states. Systems and Control Letters, 104:66$71,2017$.

[4] Georges Bastin, Jean-Michel Coron, and Brigitte d'Andréa-Novel. On Lyapunov stability of linearised Saint-Venant equations for a sloping channel. Networks and Heterogeneous Media, 4(2):177-187, 2009.

[5] Georges Bastin, Jean-Michel Coron, and Simona Oana Tamasoiu. Stability of linear densityflow hyperbolic systems under PI boundary control. Automatica J. IFAC, 53:37-42, 2015.

[6] Jean-Michel Coron, Brigitte d'Andréa-Novel, and Georges Bastin. A Lyapunov approach to control irrigation canals modeled by Saint-Venant equations. In Proceedings European Control Conference, Karlsruhe, Germany, September 1999.

[7] Jean-Michel Coron, Brigitte d'Andréa-Novel, and Georges Bastin. A strict Lyapunov function for boundary control of hyperbolic systems of conservation laws. IEEE Transactions on Automatic Control, 52(1):2-11, January 2007.

[8] Jean-Michel Coron and Hoai-Minh Nguyen. Dissipative boundary conditions for nonlinear 1-D hyperbolic systems: sharp conditions through an approach via time-delay systems. SIAM Journal on Mathematical Analysis, 47(3):2220-2240, 2015.

[9] Jean Michel Coron and Simona Oana Tamasoiu. Feedback stabilization for a scalar conservation law with PID boundary control. Chin. Ann. Math. Ser. B, 36(5):763-776, 2015.

[10] Jean-Michel Coron, Rafael Vazquez, Miroslav Krstic, and Georges Bastin. Local exponential $H^{2}$ stabilization of a $2 \times 2$ quasilinear hyperbolic system using backstepping. SIAM Journal of Control and Optimization, 51(3):2005-2035, 2013.

[11] Jonathan de Halleux, Christophe Prieur, Jean-Michel Coron, Brigitte d'Andréa-Novel, and Georges Bastin. Boundary feedback control in networks of open-channels. Automatica, 39(8):1365-1376, 2003.

[12] Joachim Deutscher. Backstepping design of robust state feedback regulators for linear $2 \times 2$ hyperbolic systems. IEEE Transactions on Automatic Control, 62(10):5240-5247, 2017.

[13] Ababacar Diagne, Georges Bastin, and Jean-Michel Coron. Lyapunov exponential stability of 1-D linear hyperbolic systems of balance laws. Automatica J. IFAC, 48(1):109-114, 2012.

[14] Valrie Dos Santos, Georges Bastin, Jean-Michel Coron, and Brigitte d'Andréa-Novel. Boundary control with integral action for hyperbolic systems of conservation laws : stability and experiments. Automatica, 44(5):1310 - 1318, 2008.

[15] Amaury Hayat and Peipei Shang. A quadratic Lyapunov function for Saint-Venant equations with arbitrary friction and space-varying slope. Preprint, 2017. 
[16] Guenter Leugering and E. J. P. Georg Schmidt. On the modelling and stabilization of flows in networks of open canals. SIAM J. Control Optim., 41(1):164-180, 2002.

[17] Xavier Litrico and Vincent Fromion. Boundary control of hyperbolic conservation laws using a frequency domain approach. Automatica J. IFAC, 45(3):647-656, 2009. 